\title{
Primary Hyperparathyroidism Masquerading Malignancy: A Case Report
}

\author{
Mubashir Maqbool Wani ${ }^{\mathrm{a}, \mathrm{c}}$, Manzoor A Halwai ${ }^{\mathrm{a}}$, Bashir A Mir ${ }^{\mathrm{a}}$, \\ Anwar Hussain ${ }^{\mathrm{a}}$, Nahida Akhter ${ }^{\mathrm{b}}$
}

\begin{abstract}
Skeletal manifestations in the form of brown tumors are rare and according to literature occur in less than $2 \%$ of patients suffering from any form of hyperparathyroidism. Such rare and multiple benign lesions may simulate a malignant neoplasm and pose a real challenge for the clinician in its differential diagnosis. We present a case that was evaluated for multiple lytic lesions with a strong suspicion of malignancy but turned out to be a case of primary hyperparathyroidism.
\end{abstract}

Keywords: Hyperparathyroidism; Multiple lytic lesions; Malignancy

\section{Case Report}

A 70 years old female Asian Indian in origin presented to our orthopedic emergency department as a case of pain right thigh area and right arm following a trivial trauma. The patient was already bed ridden and was being evaluated for multiple myeloma in a tertiary care medical oncology center.

Radiographic examination of the affected areas was done which revealed multiple lytic lesions of both proximal femurs with fracture subtrochanteric area on right side (Fig. 1) and multiple lytic lesions in right humerus with fracture

Manuscript accepted for publication May 20, 2010

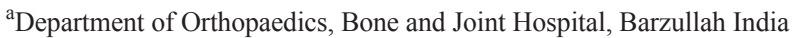
190005

${ }^{\mathrm{b}}$ Department of Anaesthesiology, Government SMHS Hospital, Srinagar India 190010

${ }^{\mathrm{c}}$ Corresponding Author: Department of Orthopaedics, Bone and Joint Hospital, Barzullah India 190005

Email: mubashirmaqboolwani@yahoo.co.in

doi:10.4021/jmc2010.07.106e in midshaft (Fig. 2). Her serum chemistry revealed significantly raised calcium of $3.73 \mathrm{mmol} / 1$ (corrected range: $2.10-2.60 \mathrm{mmol} / \mathrm{l}$ ) and alkaline phosphatase of $225 \mathrm{IU} / 1$ with a normal phosphorus level. Serum electrophoresis for ' $M$ ' band was negative. There were no urinary Bence Jones proteins and CEA markers where negative. The patient

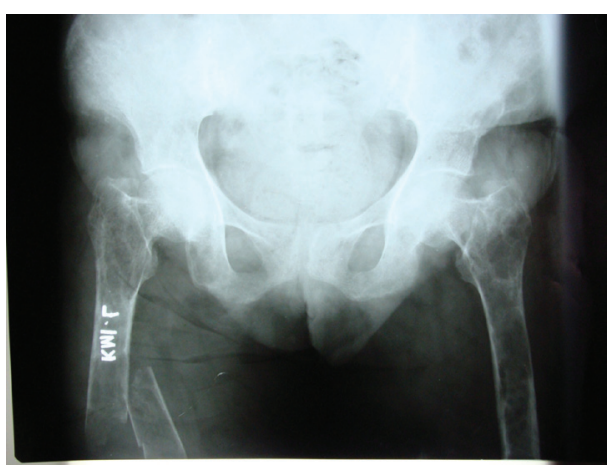

Figure 1. Plain X-ray pelvis with both femurs of a 70 year old female. Multiple lytic lesions in bilateral proximal femurs with fracture in subtrochanteric area on the right side.

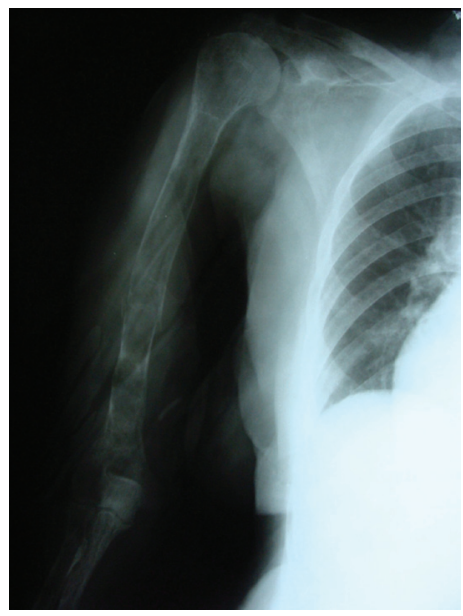

Figure 2. Plain X-ray humerus with shoulder AP view of the same patient showing Multiple lytic lesions with a pathological fracture. 
was further evaluated and it was believed that these multiple lytic lesions could be of metastatic origin. A thorough physical examination was done and a series of tests which included chest radiography, skeletal survey, abdominal ultrasound, mammography, computed tomography and bone scan were done but failed to reveal a malignant cause.

Fracture humerus in the patient was in place and was immobilized in the cast. Closed intramedullary nailing was done for her fracture subtrochanteric area. Femoral reamings were sent for histopathological examination which failed to show any evidence of malignancy. Since patient had persistent hypercalcemia, a parathyroid hormone assay was performed. This revealed a parathyroid hormone (PTH) level of $1841 \mathrm{pg} / \mathrm{ml}$ (range: 15 to $68.3 \mathrm{pg} / \mathrm{ml}$ ). Parathyroid subtraction $\mathrm{Tc}^{99 \mathrm{~m}}$ sestamibi scintigraphy showed extensive uptake in the right lower neck region consistent with parathyroid adenoma. Parathyroidectomy was performed and it was confirmed by histopathological examination that the patient had a benign parathyroid adenoma.

\section{Discussion}

Primary hyperparathyroidism means hyper functioning of one or more parathyroid glands resulting in increased parathyroid hormone levels in blood. Skeletal manifestations in the form of brown tumors are rare and according to literature occur in less than $2 \%$ of patients suffering from any form of hyperparathyroidism [1]. Such rare and multiple benign lesions may simulate a cancer and pose a real challenge for the clinician in its differential diagnosis. Lytic lesions caused by hyperparathyroidism are called brown tumors. The term "brown tumor" is a misnomer because it is not a true neoplasm. Lytic lesions in hyperparathyroidism although rare commonly arise in pelvis, ribs, extremities and other locations [2]. Diagnostic confusion arises mainly when the clinician encounters multiple lytic lesions involving different areas of the skeleton as was in our patient. There have been many cases reported describing primary hyperparathyroidism simulating malignancy [3, 4].About $90 \%$ of patients with skeletal metastases present with multiple lesions [5]. The primary tumor needs to be looked into and patient needs to be evaluated thoroughly including taking a good medical history; physical examination; routine laboratory analysis; plain radiographs of the involved bones and the chest; and the computed tomography of the chest, abdomen and pelvis. These tests did not reveal a primary tumor in our patient. Because the patient had hypercalcemia, it prompted us to go for a parathyroid hormone assay even though hypercalcemia occurs in $5-10 \%$ of all patients with advanced cancer also. To conclude, in the presence of hypercalcaemia and radiographic evidence of multiple lytic lesion primary hyperparathyroidism should always be kept in differential diagnosis and should be looked into once more common causes such as malignancy has been excluded. A high index of suspicion will lead to an early diagnosis.

\section{Competing Interests}

The authors declare that they have no competing interests.

\section{Consent}

Written informed consent was obtained from the patient for publication of this case report and accompanying images. A copy of the written consent is available for review by the Editor-in-Chief of this journal.

\section{Conflict of Interest Statement}

No benefits in any form have been or will be received from a commercial party related directly or indirectly to the subject of this manuscript

\section{Authors Contributions}

M.M. Wani designed the study, wrote the manuscript, performed literature review. M.A. Halwai, B.A. Mir, A. Hussain, NA helped in literature review and drafting the final manuscript. All authors read and approved the final manuscript.

\section{References}

1. Weatherall DJ, Ledingham JGG, Warrell DA, editors. Oxford textbook of medicine. 3rd ed. Oxford University Press; 1996. p. 1630-3.

2. Kanaan I, Ahmed M, Rifai A, Alwatban J. Sphenoid sinus brown tumor of secondary hyperparathyroidism: case report. Neurosurgery 1998;42(6):1374-1377.

3. Hsieh MC, Ko JY, Eng HL. Pathologic fracture of the distal femur in osteitis fibrosa cystica simulating metastatic disease. Arch Orthop Trauma Surg 2004;124(7):498501.

4. Chuang TC, Chang JM, Hwang SJ, Hsiao PJ, Lai YH. A patient of primary hyperparathyroidism with full-blown bone changes simulating malignancy. Kaohsiung J Med Sci 1998;14(9):584-589.

5. Tumeh SS, Kaplan WD. Clinical significance of solitary rib lesions in patients with extra skeletal malignancy. J NucI Med. 1985;26: 1140-1143. 